\title{
Intersections
}

Canadian Journal of Music

Revue canadienne de musique

\section{Laurent Feneyrou, Giordano Ferrari et Geneviève Mathon, éd. 2007. À Bruno Maderna, volume 1. Paris : Basalte. 558 p. ISBN 978-2-9526717-1-2 (couverture souple)}

\section{Yves Balmer}

Volume 29, numéro 1, 2009

URI : https://id.erudit.org/iderudit/039117ar

DOI : https://doi.org/10.7202/039117ar

Aller au sommaire du numéro

Éditeur(s)

Canadian University Music Society / Société de musique des universités canadiennes

ISSN

1911-0146 (imprimé)

1918-512X (numérique)

Découvrir la revue

Citer ce compte rendu

Balmer, Y. (2009). Compte rendu de [Laurent Feneyrou, Giordano Ferrari et Geneviève Mathon, éd. 2007. À Bruno Maderna, volume 1. Paris : Basalte. 558 p. ISBN 978-2-9526717-1-2 (couverture souple)]. Intersections, 29(1), 123-129.

https://doi.org/10.7202/039117ar

Tous droits réservés (C) Canadian University Music Society / Société de musique des universités canadiennes, 2009 lntersections

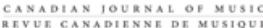

Ce document est protége par la loi sur le droit d'auteur. L'utilisation de services d'Érudit (y compris la reproduction) est assujettie à sa politique d'utilisation que vous pouvez consulter en ligne.

https://apropos.erudit.org/fr/usagers/politique-dutilisation/ 


\section{RÉFÉRENCES}

Benjamin, Walter. 1982. «Paris, capitale du XIXe siècle [1939] ». Dans Das Passagen-Werk, 60-77. Frankfurt am Main : Suhrkamp Verlag.

Dahlhaus, Carl. 1991. Nineteenth-Century Music. Berkeley : University of California Press.

Ehrhardt, Damien. 2008a. "Le salon de Liszt comme symbole du 'Nouveau Weimar' (1848-1861)». Dans Mélodies urbaines. La musique dans les villes d'Europe. XVI $-X I X^{e}$ siècles, sous la dir. de Laure Gauthier \& Mélanie Traversier, 221-239. Paris : Presses de l'Université Paris-Sorbonne.

Ehrhardt, Damien. 2008b. "Liszt, médiateur entre la France et l'Allemagne. Vers une nouvelle théorie du champ et une histoire transculturelle de la musique ». Dans Franz Liszt - Musique, médiation, interculturalité, sous la dir. de Damien Ehrhardt, 503-527. Études germaniques 63, no 3 .

Keeling, Geraldine. 1986/1987. "Liszt's Appearances in Parisian Concerts, 1824-1844 ». Liszt Society Journal 11 [1986] : 22-34 et 12 [1987] : 8-22.

Kleinertz, Rainer (dir.). 200o. Franz Liszt. Sämtliche Schriften 1: Frühe Schriften. Wiesbaden, Leipzig \& Paris : Breitkopf \& Härtel.

Mattelart, Armand, et Erik Neveu. 2008. Introduction aux Cultural Studies. Paris : La Découverte.

Rehding, Alexander. 2002. "Liszt's Musical Monuments ». 19th Century Music $26, \mathrm{n}^{\circ} 1: 52-72$.

Schmitt, Hugues. 2008. "Recital et recitatio. Réflexions autour de la performance musicale chez Liszt ». Dans Franz Liszt - Musique, médiation, interculturalité, sous la dir. de Damien Ehrhardt, 473-484. Études germaniques $63, \mathrm{n}^{\circ} 3$.

DAMIEN EHRHARDT

Laurent Feneyrou, Giordano Ferrari et Geneviève Mathon, éd. 2007. À Bruno Maderna, volume 1. Paris : Basalte. 558 p. ISBN 978-2-9526717-1-2 (couverture souple).

Si Bruno Maderna (1920-1973) est aujourd'hui considéré comme une " figure de premier plan » dans l'historiographie musicale de la seconde moitié du XX siècle, force est de constater que les livres le concernant sont encore rares, en France notamment, surtout au regard de la bibliographie consacrée à ses compatriotes Luciano Berio et Luigi Nono. La publication de la thèse de Nicolas Verzina, parue sous le titre Bruno Maderna, étude historique et critique, avait, en 2003, commencé à combler ce manque. Le présent ouvrage, premier de deux volumes collectifs, constitue une contribution remarquable et de grande ampleur à la connaissance de l'artiste : l'univers du compositeur est en effet étudié au sein d'une trentaine d'articles, de taille et de contenu variés, réunissant une pléiade de spécialistes des musiques italiennes (Borio, Baroni, De Benedictis, Dalmonte pour ne citer qu'eux). Le résultat est à la hauteur de l'ambition 
affichée par les éditeurs : proposer en français " une étude monographique, vaste synthèse de l'activité et de l'œuvre de Bruno Maderna, réunissant diverses compétences » (p. 7). Notons que le volume mélange des articles neufs avec une sélection judicieuse d'études plus anciennes, éventuellement refondues ou mises à jour par leurs auteurs, et proposées pour la première fois en français. Les trois éditeurs gravitent dans le champ des études sur le XXe siècle et ont fait du domaine italien l'une de leur spécialité : on doit à Geneviève Mathon différents articles sur Maderna ou sur des questions liées notamment à la vocalité; Laurent Feneyrou est quant à lui l'éditeur et traducteur des écrits de Giacomo Manzoni (2006) et Luigi Nono (1993, 2007), ainsi que l'auteur d'une étude sur Il canto sospeso de Nono (2002); Giordano Ferrari, spécialiste de questions de dramaturgie (2006, 2007, 2008), a écrit sur le théâtre musical d'avant-garde en Italie (2000). Après une introduction des éditeurs du volume (" Aimer Bruno Maderna ») avouant d'emblée leur " passion commune pour l'homme et le musicien que fut Bruno Maderna » (p. 6), le propos est articulé en trois pôles : les deux premiers se consacrent aux œuvres et techniques de composition mardernienne (« Dramaturgie », p. 17-237; " Euvres vocales», p. 241-317), le dernier à la personnalité, la poétique et l'esthétique du compositeur ("Sur Bruno Maderna », p. 321-457).

\section{«Dramaturgie "}

Si l'« introduction en forme d'ouverture » de Giordano Ferrari (p. 17-24) sillonne l'ensemble de la production mardernienne, montrant à la fois les différentes réalisations dramaturgiques et l'évolution de la pensée du compositeur en ce domaine, les articles sont ensuite répartis en trois groupes distincts : un premier chapitre explore les œuvres «Des Studi per "Il processo" di Franz Kafka au Satyricon ", tandis que le suivant est consacré exclusivement à " La constellation Hyperion ». Le dernier traite quant à lui de la thématique dramaturgique "À la radio, au théâtre et au cinéma ", l'ensemble montrant ainsi l'ampleur des différents terrains embrassés par le compositeur et dans lesquels il a pu exercer son esprit dramaturgique.

Les études de cas font montre d'approches très différentes : alors que Gianmario Borio étudie "La technique sérielle dans les Studi per "Il processo" di Franz Kafka» (p. 25-37) et réfléchit sur la place particulière de cette œuvre dans l'évolution du sérialisme madernien, dont les différents moments sont rappelés, l'étude suivante (" 'Ici peut-être une cadence brillante' Voyage dans le Venitian Journal », par Angela Ida De Benedictis, p. 39-68) se consacre principalement au texte de l'œuvre, de sa genèse à son utilisation par le musicien. L'analyse du Satyricon par Geneviève Mathon (p. 69-86) appréhende quant à elle l'œuvre dans son ensemble pour en donner une présentation générale. Le propos se focalise ensuite sur une œuvre phare du compositeur, Hypérion, cycle d'œuvres composées entre 1962 et 1969 et exécutées « dans des combinaisons toujours renouvelées » (p. 123). L'œuvre est présentée comme «l'un des exemples les plus importants du $\mathrm{XX}^{\mathrm{e}}$ siècle d'une dramaturgie où le livret ne développe aucune histoire, aucune intrigue : le contenu de l'œuvre est une idée, une situation 
existentielle, et non la narration d'une série d'événements » (p. 89). «Hyperion, les chemins du poète» (p. 90-96), texte de Giordano Ferrari, évoque le dialogue mardernien avec le roman épistolaire Hyperion de Hölderlin. Les trois réalisations scéniques que l'œuvre a connues du vivant de l'auteur sont présentées synthétiquement, mettant en évidence pour chacune la « vision différente de la poétique inspirée par le thème de Hyperion ». De plus, le panorama des versions de l'œuvre est utilement complété par la présentation de la quatrième version préparée pour la radio par Maderna, sous le titre Hyperion IV, versione per coro. La comparaison de ces différentes réalisations fait à la fois ressortir l'unité de l'œuvre et l'évolution de sa conception dans l'esprit de son créateur. Ferrari explore ensuite "les 'mots' de Hyperion " (p. 98-103) et met en lumière les niveaux de rapports entre texte et musique au sein de l'œuvre. Il conclut ainsi son investigation : "Maderna a utilisé le texte comme source d'inspiration, à développer en musique, comme élément sonore, et donc partie intégrante du processus musical, mais aussi comme élément de suggestion, capable d'aider l'auditeur à s'introduire dans la trame musicale de l'œuvre » (p. 103). Son propos l'amène enfin à formuler l'hypothèse qu'Hyperion puisse être la formulation théorique d'une pensée musicale que Maderna rechignait à coucher sur le papier.

Si Hyperion, "véritable recueil de partitions indépendantes ", est une œuvre ouverte aux multiples « solutions formelles » (p. 90), Gianmario Borio et Veniero Rizzardi, aux p. 123-161, insistent cependant sur « L’unité musicale de Hyperion » : "L'identité poétique de Hyperion est ancrée dans une unité essentiellement musicale, qui se vérifie sur trois plans différents : un même matériau, des procédés analogues de composition et des idées formelles [qui] sont non seulement au noyau central du cycle, c'est-à-dire à Dimensioni III et à Aria, mais aussi à l'œuvre suivante Stele per Diotima» (p. 124-125). À la faveur de nombreuses esquisses présentées en fac-simile, les auteurs livrent une chronologie de la naissance du matériau, précisément analysé, de l'œuvre à la genèse complexe.

C'est finalement la musique destinée à la radio, au théâtre et au cinéma qui est passée au filtre de l'analyse. L'opéra radiophonique de 1961, Don Perlimplin, ovvero il Trionfo dell'amore e dell'immaginazione, est étudié par Giordano Ferrari. Giorgi Mangini, auteur d'un livre sur Maderna et le cinéma (1998), offre un article d'une grande précision documentaire : sa contribution (p. 229-237) consiste en une « filmographie commentée » qui présente onze notices synthétiques avec bibliographie pour chacun des films auxquels Maderna a participé, comme compositeur de la musique de film ou comme chef d'orchestre d'un opéra filmé (Wozzeck) avec une fonction éventuelle d'arrangeur (Lohengrin). Maurizio Romito, dans son article intitulé " De l'écran à la scène : Les musiques de Maderna pour la télévision et le théâtre » (p. 177-224), propose des fiches signalétiques fort circonstanciées sur chacune des productions du compositeur dans ces domaines, le propos étant accompagné d'annexes relevant la localisation dans le film de chaque composition musicale, de sa durée et sa nomenclature, et, le cas échéant, des textes utilisés. Est en outre intégré dans cette section le témoignage de l'écrivain Rebecca Rass, dont l'ouvrage de 1969 From A to $Z$ fut l'objet d'une " production multimédia d'art télévisuel » pour 
laquelle elle demanda à Maderna de créer la partie musicale. Une année après cette expérience, Maderna composait son opéra éponyme.

\section{«Cuvres vocales"}

La seconde partie du livre, consacrée aux « Euvres vocales » du compositeur, débute par une introduction de Raymond Fearn (biographe de Maderna (1990) et auteur en 1997 d'un Italian Opera Since 1945) traitant des rapports du compositeur à la voix. Il rappelle l'importance de la figure de Dallapiccola pour la génération des compositeurs italiens de l'après-guerre - notamment en raison du rôle central de la voix dans son œuvre-, puis liste les œuvres de Maderna où la voix joue un rôle déterminant (p. 243); il juge toutefois que « c'est certainement dans son œuvre instrumentale que nous trouvons le plus haut degré d'innovation et d'inspiration » (p. 245). Les trois articles suivants se consacrent à l'étude d'œuvres précises. Luca Conti analyse les Tre liriche greche dans leur rapport au dodécaphonisme notamment (p. 249-262); N. Verzina observe les Vier Briefe ("La dialectique histoire-composition dans la musique italienne du second après-guerre : Sérialisme et engagement dans la Kranichsteiner Kammerkantate (Vier Briefe) », p. 263-286), autant du point de vue des techniques de composition, plus particulièrement la "mutation sérielle " propre à Maderna (expliquée aux p. 272-274), que du point de vue de l'œuvre appartenant à la «culture engagée » italienne des années 1950. A. I. De Benedictis consacre ensuite son propos à Ausstrahlung (p. 287-317) et oriente le lecteur dans cette œuvre labyrinthique, dont la « structure musicale, par sa complexité, peut être considérée comme une summa des différentes expériences inhérentes à l'ambiguïté textuelle, un compendium des possibles configurations micro- et macroformelles d'une composition mobile (ou en "mouvement"), conditionnée par les poétiques du montage, de l'indétermination et du fragment» (p. 291).

\section{"Sur Bruno Maderna "}

Cette dernière partie de l'ouvrage s'ouvre sur cinq témoignages. Le premier est une traduction d'un texte d'Ulrich Mosch (écrit pour la réédition de 1999 du premier livre en italien sur Maderna, Maderna Musicista europeo de Massimo Mila) dans lequel sont notamment analysées les raisons pour lesquelles Maderna n'aurait pas, aux premiers moments de l'historiographie consacrée à la musique de l'avant-garde de la seconde moitié du $\mathrm{XX}^{\mathrm{e}}$ siècle, été considéré comme un compositeur de premier ordre, au détriment d'autres figures comme celles de Boulez et de Stockhausen. À la suite de ce texte, les éditeurs ont reproduit le texte d'introduction du livre précédemment évoqué de Mila (1976), qui permet de prendre le pouls de la recherche madernienne au moment où cette publication a vu le jour. C'est ensuite la critique Martine Cadieu (proche de plusieurs compositeurs italiens dont Nono et Dallapiccola) qui confie ses souvenirs de Bruno Maderna, rencontré à Amsterdam, Venise, Danaueschingen ou encore Royan. Le compositeur Konrad Boehmer livre très directement son expérience d'assistant de Bruno Maderna au studio électronique de la radio de Cologne pour réaliser les parties de la bande d'Intolleranza 1960 de Nono. Il y dévoile 
de nombreux souvenirs concernant les positions politiques du compositeur ou ses opinions musicales, par exemple celle-ci : «Il considérait les maîtres de Cologne comme une bande de révolutionnaires incultes. Son attitude envers Stockhausen fut extrêmement critique. Il avait horreur de cette posture de grand prêtre que Stockhausen avait déjà adoptée, et il nourrissait des soupçons profonds envers la conception de Cologne d'un sérialisme hors ou au-dessus de toute sensualité » (p. 347). La série des témoignages se clôt par un texte de Nono, "En souvenir de deux musiciens » (p. 355-357), hommage double, à Maderna d'une part et à Malipiero d'autre part, qui lui avait « conseillé de faire la connaissance » (p. 355) du premier.

Le second chapitre de la partie "Sur Bruno Maderna " traite de "poétique et esthétique ». Cinq études y prennent place. Luigi Pestalozza s'intéresse aux "Années milanaises» de Bruno Maderna (p. 361-377). Cet article est présenté par les éditeurs comme " essentiel » car " récusant toute assimilation postmoderne de la multiplicité des styles et des pratiques maderniens, à la faveur d'un décentrement démocratique et galiléen » (p. 9). Mario Baroni signe l'article « Poétiques, comportements, style de vie : Est-il possible que nous ayons quelque chose en commun ?" (p. 379-387). Il y soutient l'idée que la poétique de Maderna présente une « contradiction profonde entre les exigences de l'histoire musicale de son époque et celles de [1]a vocation humaine [du compositeur] " (p. 386). L'auteur propose alors de trouver les " véritables sources de sa poétique " dans les images de répétition du Concerto $n^{\circ}{ }_{3}$ pour hautbois et orchestre, œuvre dans laquelle Maderna " compose et interprète " dans le même temps. Rossana Dalmonte, qui a signé l'article sur Bruno Maderna paru dans le New Grove de 2001 (repris dans le Grove Music Online), offre une étude intitulée "Maderna et la musique : notes de poétique », principalement fondée sur les écrits du compositeur (p. 389-404). Giovanni Morelli écrit « La charge des quodlibet", article sous-titré "Notes sur la typologie idéale d'une 'nouvelle école vénitienne' à l'usage d'une lecture croisée des œuvres de Maderna, Nono et Malipiero » (p. 405-435). Enfin, Laurent Feneyrou dresse un portrait de Bruno Maderna en "musicien de la vie ", montrant chez le compositeur "l'unité, sinon la synonymie de l'œuvre et de la vie. [ ... ] Où les modes de vie suscitent des œuvres, créatrices de conduites » (p. 438).

La fin du livre apporte une documentation précieuse qui servira les recherches futures consacrées à Maderna. Cette documentation présente des extraits significatifs de la correspondance (p. 459-517), classée chronologiquement, et dont l'appareil critique permet de saisir avec précision le contexte. La chronologie qui suit liste par années (ou presque) les événements de la vie de Maderna, de telle sorte qu'y sont mêlés vie professionnelle et événements privés. Le degré de précision varie d'un événement à l'autre mais offre, au sein de ce volume d'études, une contribution biographique utile au lecteur peu familier avec le compositeur. Le livre se clôt sur une riche bibliographie, présentant, d'un côté, les écrits sur Maderna (les auteurs ont malheureusement choisi de ne pas insérer les travaux universitaires, y compris les thèses de doctorat), et inventoriant, de l'autre, avec précision les écrits de Maderna (séparant les «Articles et écrits publiés ", les "Conférences, notes et écrits publiés de manière posthume », les 
« Entretiens et conversations publiés », la « Correspondance publiée », enfin la "Liste chronologique des notices et programmes de concert »).

En dépit d'un problème d'impression affectant la page 19, la présentation du livre reste fort soignée. Si la majeure partie des fac-simile d'esquisses autographes est de très bonne qualité à la lecture, quelques-uns auraient gagné à être également présentés en transcription de manière à obtenir plus de lisibilité (p. 149 par exemple). De même, plusieurs extraits musicaux manuscrits sont peu lisibles; leur saisie aurait pu offrir un meilleur confort de lecture (p. 107 et p. 109 notamment).

Il est évident que ce volume contribue de façon magistrale à la connaissance du compositeur, ce dont témoigne, par ailleurs, l'accueil critique de l'ouvrage (Prix des Muses 2008 dans la catégorie " Document musicologique "; "Coup de cœur musique contemporaine printemps 2008 "de l'Académie Charles Cros). Présentant de nombreux aspects poétiques, esthétiques et analytiques sous les plumes d'auteurs de renom proposant des méthodologies variées, le livre expose la densité de l'œuvre et la puissance de la pensée du compositeur, tout en invitant, par la multiplicité de pistes ouvertes, à de futures recherches.

La publication sera complète lors de la parution du second volume de cette entreprise musicologique consacrée à Maderna. Le prochain tome aura pour objet " l'œuvre instrumentale et électronique, et [le] Maderna transcripteur, amateur de jazz et chef d'orchestre »; il inclura également une partie documentaire : catalogue et discographie du compositeur et de l'interprète.

\section{RÉFÉRENCES}

Dalmonte, Rossana. "Bruno Maderna ». Grove Music Online [consulté le 19 août 2009].

Fearn, Raymond. 1990. Bruno Maderna. Chur : Harwood Academic Publishers.

— 1997. Italian Opera since 1945. Amsterdam : Harwood Academic Publishers.

Feneyrou, Laurent. 2002. "Il canto sospeso" de Luigi Nono : musique et analyse. Paris : Michel de Maule.

Ferrari, Giordano. 200o. Les débuts du théâtre musical d'avant-garde en Italie : Berio, Evangelisti, Maderna. Paris : L'Harmattan.

- (dir.). 2006. L'opéra éclaté : la dramaturgie musicale entre 1969 et 1984. Paris : L'Harmattan.

(dir.). 2007. La musique et la scène : l'écriture musicale et son expression scénique au $X X^{e}$ siècle. Paris : L'Harmattan.

(dir.). 2008. La parole sur scène, voix, texte, signifié. Paris : L'Harmattan.

Manzoni, Giacomo. 2006. Écrits. Textes réunis, traduits, annotés et présentés par Laurent Feneyrou. Paris : Basalte.

Nono, Luigi. 1993. Écrits. Réunis, présentés et annotés par Laurent Feneyrou; traduction sous la dir. de Laurent Feneyrou. Paris : C. Bourgois.

- 2007. Écrits. Nouvelle édition française de Laurent Feneyrou, basée sur l'édition italienne d'Angela Ida De Benedictis et de Veniero Rizzardi; 
textes traduits de l'italien et de l'allemand par Laurent Feneyrou. Genève : Contrechamps.

Mangini, Giorgio. 1998. Le esperienze cinematografiche di Bruno Maderna. Venise : Videoteca Pasinetti.

Mila, Massimo. 1976. Maderna Musicista europeo. Turin : Einaudi.

Verzina, Nicolas. 2003. Bruno Maderna, étude historique et critique. Paris :

L'Harmattan.

Yves BALMER

Anna Hoefnagels and Gordon E. Smith, eds. 2007. Folk Music, Traditional Music, Ethnomusicology: Canadian Perspectives, Past and Present. Newcastle, UK: Cambridge Scholars Publishing. 274 pp. (b/w photos, model diagrams). ISBN 978-1-84718-366-8.

This book is a collection of papers selected from the proceedings at the fiftieth anniversary meeting of the Canadian Society for Traditional Music (CSTM). The editors of this collection, Anna Hoefnagels and Gordon E. Smith, envisaged a volume that "showcases the diversity of music research currently being conducted by folk and traditional music specialists, ethnomusicologists and practicing musicians in Canada" (xi). The chapters are organized into thematic sections: regional and historical perspectives in Canadian ethnomusicology; issues ethnomusicologists are facing in their practice; music performers, traditions, and musical expressions; First Peoples' music; and, in a final section reflecting on the CSTM and writing about folk and traditional music scholarship, several chapters offer a dialogue on the society's past and some possible future directions.

Hoefnagels and Smith provide an introduction that contextualizes the papers in the collection within a retrospective of the CSTM. Here, they express their hope that the volume will serve as an important contribution to the literature on ethnomusicology and folklore studies in Canada, for academics and music enthusiasts. Hoefnagels and Smith also pay homage to important volumes on Canadian music studies, notably Diamond and Witmer's Canadian Music (1994). This well-known volume seems to be the theoretical forbear of the new collection, for its diversity is not in conventional Canadian terms (a given ethnicity or region) but of issues, themes, and problems. While the new collection is limited to work presented at the CSTM meeting, it includes a range of contemporary ethnomusicological scholarship on timely topics.

The writing of scholars at various points in their careers is included in this volume - another valuable aspect of its particular diversity, providing evocative juxtapositions of methods, aims, and topics. For instance, two papers by junior scholars (Sparling and McDonald) address the concept and transmission of contemporary "folk" music, and sit next to a paper by a more senior scholar that examines the historical collection of yet another kind of "folk" music (Gregory). Taken together, these raise exciting questions about the construction, practice, 